J. Indones. Math. Soc.

Vol. 19, No. 2 (2013), pp. 79-87.

\title{
ON STRONG AND WEAK CONVERGENCE IN $n$-HILBERT SPACES
}

\author{
Agus L. Soenjaya \\ Department of Mathematics, National University of Singapore, Singapore \\ agus.leonardi@nus.edu.sg
}

\begin{abstract}
We discuss the concepts of strong and weak convergence in $n$-Hilbert spaces and study their properties. Some examples are given to illustrate the concepts. In particular, we prove an analogue of Banach-Saks-Mazur theorem and Radon-Riesz property in the case of $n$-Hilbert space.
\end{abstract}

Key words: Strong and weak convergence, $n$-Hilbert space.

\begin{abstract}
Abstrak. Makalah ini menjelaskan konsep konvergensi kuat dan lemah dalam ruang $n$-Hilbert dan mempelajari sifat-sifatnya. Beberapa contoh diberikan untuk menjelaskan konsep-konsep tersebut. Khususnya, teorema analog dari Banach-SaksMazur dan sifat Radon-Riesz dibuktikan untuk kasus ruang $n$-Hilbert..
\end{abstract}

Kata kunci: Konvergensi kuat dan lemah, ruang $n$-Hilbert.

\section{INTRODUCTION AND PRELIMINARIES}

The notion of $n$-normed spaces was introduced by Gähler ([4]) as a generalization of normed spaces. It was initially suggested by the area function of a triangle determined by a triple in Euclidean space. The corresponding theory of $n$-inner product spaces was then established by Misiak ([10]). Since then, various aspects of the theory have been studied, for instance the study of Mazur-Ulam theorem and Aleksandrov problem in $n$-normed spaces are done in [1, 2], the study of operators in $n$-Banach space is done in [5,11], and many others.

In this paper, we will generalize the notion of weak convergence in Hilbert space to the case of $n$-Hilbert space and study its properties. In particular, we will expand on the results in [6]. We will also give an analogue of Radon-Riesz property (on conditions relating strong and weak convergence) in the case of $n$-Hilbert space. Furthermore, an analogue of the well-known Banach-Saks-Mazur theorem (on the

2000 Mathematics Subject Classification: Primary 40A05; Secondary 46C99.

Received: 17-07-2012, revised: 31-03-2013, accepted: 05-06-2013. 
strong convergence of a convex combination of a weakly convergent sequence) will be given.

We begin with some preliminary results. Let $X$ be a real vector space with $\operatorname{dim}(X) \geq n$, where $n$ is a positive integer. We allow $\operatorname{dim}(X)$ to be infinite. A real-valued function $\|\cdot, \ldots, \cdot\|: X^{n} \rightarrow \mathbb{R}$ is called an $n$-norm on $X^{n}$ if the following conditions hold:

(1) $\left\|x_{1}, \ldots, x_{n}\right\|=0$ if and only if $x_{1}, \ldots, x_{n}$ are linearly dependent;

(2) $\left\|x_{1}, \ldots, x_{n}\right\|$ is invariant under permutations of $x_{1}, \ldots, x_{n}$;

(3) $\left\|\alpha x_{1}, x_{2}, \ldots, x_{n}\right\|=|\alpha|\left\|x_{1}, x_{2}, \ldots, x_{n}\right\|$ for all $\alpha \in \mathbb{R}$ and $x_{1}, \ldots, x_{n} \in X$;

(4) $\left\|x_{0}+x_{1}, x_{2}, \ldots, x_{n}\right\| \leq\left\|x_{0}, x_{2}, \ldots, x_{n}\right\|+\left\|x_{1}, x_{2}, \ldots, x_{n}\right\|$, for all $x_{0}, x_{1}, \ldots, x_{n} \in X$.

The pair $(X,\|\cdot, \ldots, \cdot\|)$ is then called an $n$-normed space. It also follows from the definition that an $n$-norm is always non-negative.

Let $X$ be a real vector space with $\operatorname{dim}(X) \geq n$, where $n$ is a positive integer. A real-valued function $\langle\cdot, \cdot \mid \cdot, \ldots, \cdot\rangle: X^{n+1} \rightarrow \mathbb{R}$ is called an $n$-inner product on $X$ if the following conditions hold:

(1) $\left\langle z_{1}, z_{1} \mid z_{2}, \ldots, z_{n}\right\rangle \geq 0$, with equality if and only if $z_{1}, z_{2}, \ldots, z_{n}$ are linearly dependent;

(2) $\left\langle z_{1}, z_{1} \mid z_{2}, \ldots, z_{n}\right\rangle=\left\langle z_{i_{1}}, z_{i_{1}} \mid z_{i_{2}}, \ldots, z_{i_{n}}\right\rangle$ for every permutation $\left(i_{1}, \ldots, i_{n}\right)$ of $(1, \ldots, n)$;

(3) $\left\langle x, y \mid z_{2}, \ldots, z_{n}\right\rangle=\left\langle y, x \mid z_{2}, \ldots, z_{n}\right\rangle$;

(4) $\left\langle\alpha x, y \mid z_{2}, \ldots, z_{n}\right\rangle=\alpha\left\langle x, y \mid z_{2}, \ldots, z_{n}\right\rangle$ for every $\alpha \in \mathbb{R}$;

(5) $\left\langle x+x^{\prime}, y \mid z_{2}, \ldots, z_{n}\right\rangle=\left\langle x, y \mid z_{2}, \ldots, z_{n}\right\rangle+\left\langle x^{\prime}, y \mid z_{2}, \ldots, z_{n}\right\rangle$.

The pair $(X,\langle\cdot, \cdot \mid \cdot, \ldots, \cdot\rangle)$ is then called an $n$-inner product space.

Observe that any inner product space $(X,\langle\cdot, \cdot\rangle)$ can be equipped with the standard n-inner product:

$$
\left\langle x, y \mid z_{2}, \ldots, z_{n}\right\rangle:=\left|\left(\begin{array}{cccc}
\langle x, y\rangle & \left\langle x, z_{2}\right\rangle & \cdots & \left\langle x, z_{n}\right\rangle \\
\left\langle z_{2}, y\right\rangle & \left\langle z_{2}, z_{2}\right\rangle & \cdots & \left\langle z_{2}, z_{n}\right\rangle \\
\vdots & \vdots & \ddots & \vdots \\
\left\langle z_{n}, y\right\rangle & \left\langle z_{n}, z_{2}\right\rangle & \cdots & \left\langle z_{n}, z_{n}\right\rangle
\end{array}\right)\right|
$$

where $|A|$ denotes the determinant of $A$.

In that case, the induced standard n-norm on $X$ is given by

$$
\left\|x_{1}, \ldots, x_{n}\right\|_{S}:=\sqrt{\operatorname{det}\left[\left\langle x_{i}, x_{j}\right\rangle\right]}
$$

Note that the value of $\left\|x_{1}, \ldots, x_{n}\right\|_{S}$ is just the volume of the $n$-dimensional parallelepiped spanned by $x_{1}, \ldots, x_{n}$.

Further examples and results on $n$-normed space can be found in $[8,9]$. In particular, for the standard case, completeness in the norm is equivalent to that in the induced standard $n$-norm.

Every $n$-inner product space is an $n$-normed space with the induced $n$-norm:

$$
\left\|x_{1}, \ldots, x_{n}\right\|:=\left\langle x_{1}, x_{1} \mid x_{2}, \ldots, x_{n}\right\rangle^{1 / 2}
$$


An analogue of Cauchy-Schwarz inequality also holds for $n$-inner product space, i.e. for all $x, y, z_{2}, \ldots, z_{n} \in X$, we have

$$
\left|\left\langle x, y \mid z_{2}, \ldots, z_{n}\right\rangle\right| \leq\left\|x, z_{2}, \ldots, z_{n}\right\|\left\|y, z_{2}, \ldots, z_{n}\right\|
$$

The following definitions are taken and inspired from [12].

Definition 1.1. A sequence $\left\{x_{k}\right\}$ in an $n$-normed space $(X,\|\cdot, \ldots, \cdot\|)$ is said to converge to $x \in X$ if $\left\|x_{k}-x, z_{2}, \ldots, z_{n}\right\| \rightarrow 0$ as $k \rightarrow \infty$ for all $z_{2}, \ldots, z_{n} \in X$.

Definition 1.2. A sequence $\left\{x_{k}\right\}$ in an n-normed space $(X,\|\cdot, \ldots, \cdot\|)$ is a Cauchy sequence if $\left\|x_{k}-x_{l}, z_{2}, \ldots, z_{n}\right\| \rightarrow 0$ as $k, l \rightarrow \infty$ for all $z_{2}, \ldots, z_{n} \in X$.

Definition 1.3. If every Cauchy sequence in an $n$-normed space $(X,\|\cdot, \ldots, \cdot\|)$ converges to an $x \in X$, then $X$ is said to be complete. A complete $n$-inner product space is called an $n$-Banach space. A complete $n$-inner product space is called an $n$-Hilbert space.

\section{STRONG AND WEAK CONVERGENCE}

In this section, we will consider the notions of strong and weak convergence in $n$-Hilbert space. The notion of (strong) convergence in 2-normed space has been studied extensively in [12]. Here, we will focus more on the weak convergence and the relationships between the two concepts. Let $(X,\langle\cdot, \cdot \mid \cdot, \ldots, \cdot\rangle)$ be an $n$-Hilbert space and $\|\cdot, \ldots, \cdot\|$ be the induced $n$-norm.

Definition 2.1 (Strong convergence). A sequence $\left(x_{k}\right)$ in $X$ is said to converge strongly to a point $x \in X$ if $\left\|x_{k}-x, z_{2}, \ldots, z_{n}\right\| \rightarrow 0$ as $k \rightarrow \infty$ for every $z_{2}, \ldots, z_{n} \in$ $X$. In this case, we write $x_{k} \rightarrow x$.

Definition 2.2 (Weak convergence). A sequence $\left(x_{k}\right)$ in $X$ is said to converge weakly to a point $x \in X$ if $\left\langle x_{k}-x, y \mid z_{2}, \ldots, z_{n}\right\rangle \rightarrow 0$ as $k \rightarrow \infty$ for every $y, z_{2}, \ldots, z_{n} \in X$. In this case, we write $x_{k} \rightarrow x$.

The following proposition is immediate from the definition.

Proposition 2.3. If $\left(x_{k}\right)$ and $\left(y_{k}\right)$ converges strongly (resp. weakly) to $x$ and $y$ respectively, then $\left(\alpha x_{k}+\beta y_{k}\right)$ converges strongly (resp. weakly) to $\alpha x+\beta y$.

Here we mention some of the basic properties, the proofs of which can be found in [6].

Proposition 2.4 (Continuity). The following results hold:

(1) The n-norm is continuous in each variable.

(2) The n-inner product is continuous in the first two variables.

Proposition 2.5. If $\left(x_{k}\right)$ converges strongly (resp. weakly) to $x$ and $x^{\prime}$, then $x=x^{\prime}$.

Note that strong convergence implies weak convergence.

Proposition 2.6. If $\left(x_{k}\right)$ converges strongly to $x$, then it converges weakly to $x$. 
Proof. Refer to [6].

However, the converse is not true in general. The following highlights some of the way a sequence can fail to converge strongly.

Example 2.7. Let $X=L^{2}[0,1]$ which is a Hilbert space with the usual inner product. Equip $X$ with the standard 2-inner product. Define a sequence $\left(f_{n}\right)$ by $f_{n}(x)=\sin n \pi x$. Then for all $g, h \in X$,

$$
\begin{aligned}
\left\langle f_{n}, g \mid h\right\rangle & =\left\langle f_{n}, g\right\rangle\langle h, h\rangle-\left\langle f_{n}, h\right\rangle\langle h, g\rangle \\
& \leq\left|\left(\int_{0}^{1} g(x) \sin n \pi x d x\right)\|h\|_{2}^{2}\right|+\left|\left(\int_{0}^{1} h(x) \sin n \pi x d x\right)\|h\|_{2}\|g\|_{2}\right|
\end{aligned}
$$

so that $f_{n} \rightarrow 0$, where we used the Riemann-Lebesgue lemma. However,

$$
\left\|f_{n}, h\right\|=\left(\left\|f_{n}\right\|_{2}^{2}\|h\|_{2}^{2}-\left\langle f_{n}, h\right\rangle^{2}\right)^{1 / 2}
$$

As $n \rightarrow \infty,\left\|f_{n}, h\right\| \rightarrow \frac{1}{\sqrt{2}}\|h\|_{2}$, which is not zero as long as $h \neq 0$ a.e., showing that $f_{n}$ does not converge strongly to the zero function.

Example 2.8. Let $(X,\langle\cdot, \cdot\rangle)$ be a separable infinite-dimensional Hilbert space with $\left(e_{k}\right)_{k=1}^{\infty}$ as an orthonormal basis. Equip $X$ with the standard $n$-inner product. In [6], it is proven that $\left(e_{k}\right)$ converges weakly, but not strongly to 0.

Remark 2.9. More generally, if $X$ is a separable Hilbert space and $\left\{\phi_{k}\right\}$ is an orthonormal sequence in $X$. Then $\phi_{k} \rightarrow 0$ in the induced standard $n$-inner product.

Example 2.10. Let $X=L^{2}(\mathbb{R})$, equipped with the standard 2-inner product. Define a sequence $\left(f_{n}\right)$ by $f_{n}(x)=\chi_{(n, n+1)}(x)$, where $\chi$ is the characteristic function. Then one can check that $\left(f_{n}\right)$ converges weakly, but not strongly, to zero in $X$.

Remark 2.11. In [6], it is observed that in standard, finite-dimensional $n$-Hilbert spaces, the notions of strong and weak convergence are equivalent.

We will now give an extension of Radon-Riesz property for $n$-Hilbert space.

Theorem 2.12. If $x_{k} \rightarrow x$, then

$$
\left\|x, z_{2}, \ldots, z_{n}\right\| \leq \liminf _{k \rightarrow \infty}\left\|x_{k}, z_{2}, \ldots, z_{n}\right\|
$$

If, in addition,

$$
\lim _{k \rightarrow \infty}\left\|x_{k}, z_{2}, \ldots, z_{n}\right\|=\left\|x, z_{2}, \ldots, z_{n}\right\|
$$

for all $z_{2}, \ldots, z_{n} \in X$, then $x_{k} \rightarrow x$.

Proof. Using weak convergence of $\left(x_{k}\right)$ and Cauchy-Schwarz inequality,

$$
\begin{aligned}
\left\|x, z_{2}, \ldots, z_{n}\right\|^{2}=\left\langle x, x \mid z_{2}, \ldots, z_{n}\right\rangle & =\lim _{k \rightarrow \infty}\left\langle x, x_{k} \mid z_{2}, \ldots, z_{n}\right\rangle \\
& \leq\left\|x, z_{2}, \ldots, z_{n}\right\| \liminf _{k \rightarrow \infty}\left\|x_{k}, z_{2}, \ldots, z_{n}\right\|
\end{aligned}
$$

proving (5). Next, by expanding the $n$-norm, $\left\|x_{k}-x, z_{2}, \ldots, z_{n}\right\|^{2}=\left\|x_{k}, z_{2}, \ldots, z_{n}\right\|^{2}-2\left\langle x_{k}, x \mid z_{2}, \ldots, z_{n}\right\rangle+\left\|x, z_{2}, \ldots, z_{n}\right\|^{2} \rightarrow 0$ 
using the assumptions given. Hence $x_{k} \rightarrow x$.

Next, we give an analogue of Banach-Saks-Mazur theorem for the case of $n$-Hilbert spaces.

Theorem 2.13. If $x_{k} \rightarrow x$ in $X$ and

$$
\lim _{m \rightarrow \infty} \frac{1}{m^{2}} \sum_{i=1}^{m}\left\|x_{i}-x, z_{2}, \ldots, z_{n}\right\|^{2}=0
$$

for all $z_{2}, \ldots, z_{n} \in X$, then there exists a sequence $\left(y_{k}\right)$ of finite convex combinations of $\left(x_{k}\right)$ such that $y_{k} \rightarrow x$ (strongly).

Proof. Replacing $x_{k}$ by $x_{k}-x$, we may assume $x_{k} \rightarrow 0$. Pick $k_{1}=1$ and choose $k_{2}>k_{1}$ such that $\left\langle x_{k_{1}}, x_{k_{2}} \mid z_{2}, \ldots, z_{n}\right\rangle \leq 1$ for all $z_{2}, \ldots, z_{n}$. Inductively, given $k_{1}, \ldots, k_{m}$, pick $k_{m+1}>k_{m}$ such that

$$
\left|\left\langle x_{k_{1}}, x_{k_{m+1}} \mid z_{2}, \ldots, z_{n}\right\rangle \leq \frac{1}{k}, \ldots,\right|\left\langle x_{k_{m}}, x_{k_{m+1}} \mid z_{2}, \ldots, z_{n}\right\rangle \leq \frac{1}{k}
$$

which is possible since by the weak convergence of $\left(x_{k}\right),\left\langle x_{k_{i}}, x_{k} \mid z_{2}, \ldots, z_{n}\right\rangle \rightarrow 0$ as $k \rightarrow \infty$ for $1 \leq i \leq m$. Let

$$
y_{m}:=\frac{1}{m}\left(x_{k_{1}}+\ldots+x_{k_{m}}\right)
$$

Then we have

$$
\begin{aligned}
\left\|y_{m}, z_{2}, \ldots, z_{n}\right\|^{2} & =\frac{1}{m^{2}} \sum_{i=1}^{m}\left\|x_{n_{i}}, z_{2}, \ldots, z_{n}\right\|^{2}+\frac{2}{m^{2}} \sum_{j=1}^{m} \sum_{i=1}^{j-1}\left\langle x_{n_{i}}, x_{n_{j}} \mid z_{2}, \ldots, z_{n}\right\rangle \\
& \leq \frac{1}{m^{2}} \sum_{i=1}^{m}\left\|x_{n_{i}}, z_{2}, \ldots, z_{n}\right\|^{2}+\frac{2}{m^{2}} \sum_{j=1}^{m} \sum_{i=1}^{j-1} \frac{1}{j-1} \\
& =\frac{1}{m^{2}} \sum_{i=1}^{m}\left\|x_{n_{i}}, z_{2}, \ldots, z_{n}\right\|^{2}+\frac{2}{m}
\end{aligned}
$$

so that $y_{m} \rightarrow 0$ strongly as $m \rightarrow \infty$ as required.

Corollary 2.14. Let $X$ be an Hilbert space equipped with the standard $n$-inner product. Suppose $x_{k} \rightarrow x$ in $X$ and $\left\|x_{i}\right\|<M$ for all $i$, where $M$ is a constant and $\|\cdot\|$ is the norm induced by the inner product on $X$. Then there exists a sequence $\left(y_{k}\right)$ of finite convex combinations of $\left(x_{k}\right)$ such that $y_{k} \rightarrow x$ (strongly).

Proof. It suffices to check that (6) holds in this case. Clearly, $\left\|x_{i}-x\right\|^{2}$ is also bounded in norm, say $\left\|x_{i}-x\right\|^{2}<M^{\prime}$. By Hadamard's inequality,

$$
\frac{1}{m^{2}} \sum_{i=1}^{m}\left\|x_{i}-x, z_{2}, \ldots, z_{n}\right\|^{2} \leq \frac{M^{\prime}\left\|z_{2}\right\|^{2} \ldots\left\|z_{n}\right\|^{2}}{m} \rightarrow 0
$$

as $m \rightarrow \infty$ for all $z_{2}, \ldots, z_{n} \in X$, hence the statement is proven. 


\section{APPLICATIONS}

In this section, we apply the theorems deduced earlier to $L^{2}$-space, $L^{2}(X, \mu)$, where $(X, \mu)$ is a measure space, equipped with the usual inner product

$$
\langle f, g\rangle=\int_{X} f(x) g(x) d \mu(x)
$$

We then equip $L^{2}(X)$ with the standard $n$-inner product. Note that when $n=1$, the following reduce to the familiar cases. Subsequently, $|A|=\operatorname{det}(A)$.

Proposition 3.1. Let $f_{k} \in L^{2}(X, \mu), k=1,2, \ldots$, be such that

$$
\lim _{k \rightarrow \infty}\left|\left(\begin{array}{cccc}
\int_{X} f_{k}^{2} d \mu & \int_{X} f_{k} h_{2} d \mu & \cdots & \int_{X} f_{k} h_{n} d \mu \\
\int_{X} h_{2} f_{k} d \mu & \int_{X} h_{2}^{2} d \mu & \cdots & \int_{X} h_{2} h_{n} d \mu \\
\vdots & \vdots & \ddots & \vdots \\
\int_{X} h_{n} f_{k} d \mu & \int_{X} h_{n} h_{2} d \mu & \cdots & \int_{X} h_{n}^{2} d \mu
\end{array}\right)\right|=0
$$

for all $h_{2}, \ldots, h_{n} \in L^{2}(X, \mu)$. Then

$$
\lim _{k \rightarrow \infty}\left|\left(\begin{array}{cccc}
\int_{X} f_{k} g d \mu & \int_{X} f_{k} h_{2} d \mu & \cdots & \int_{X} f_{k} h_{n} d \mu \\
\int_{X} h_{2} g d \mu & \int_{X} h_{2}^{2} d \mu & \cdots & \int_{X} h_{2} h_{n} d \mu \\
\vdots & \vdots & \ddots & \vdots \\
\int_{X} h_{n} g d \mu & \int_{X} h_{n} h_{2} d \mu & \cdots & \int_{X} h_{n}^{2} d \mu
\end{array}\right)\right|=0
$$

for all $g, h_{2}, \ldots, h_{n} \in L^{2}(X, \mu)$.

Proof. This follows from Proposition 2.6.

Proposition 3.2. Let $f_{k} \in L^{2}(X, \mu), k=1,2, \ldots$, be such that

$$
\lim _{k \rightarrow \infty}\left|\left(\begin{array}{cccc}
\int_{X}\left(f_{k}-f\right) g d \mu & \int_{X}\left(f_{k}-f\right) h_{2} d \mu & \cdots & \int_{X}\left(f_{k}-f\right) h_{n} d \mu \\
\int_{X} h_{2} g d \mu & \int_{X} h_{2}^{2} d \mu & \cdots & \int_{X} h_{2} h_{n} d \mu \\
\vdots & \vdots & \ddots & \vdots \\
\int_{X} h_{n} g d \mu & \int_{X} h_{n} h_{2} d \mu & \cdots & \int_{X} h_{n}^{2} d \mu
\end{array}\right)\right|=0
$$

for all $g, h_{2}, \ldots, h_{n} \in L^{2}(X, \mu)$. Then

$$
\begin{array}{r}
\left|\left(\begin{array}{cccc}
\int_{X} f^{2} d \mu & \int_{X} f h_{2} d \mu & \cdots & \int_{X} f h_{n} d \mu \\
\int_{X} h_{2} f d \mu & \int_{X} h_{2}^{2} d \mu & \cdots & \int_{X} h_{2} h_{n} d \mu \\
\vdots & \vdots & \ddots & \vdots \\
\int_{X} h_{n} f d \mu & \int_{X} h_{n} h_{2} d \mu & \cdots & \int_{X} h_{n}^{2} d \mu
\end{array}\right)\right| \\
\leq \liminf _{k \rightarrow \infty}\left|\left(\begin{array}{cccc}
\int_{X} f_{k}^{2} d \mu & \int_{X} f_{k} h_{2} d \mu & \cdots & \int_{X} f_{k} h_{n} d \mu \\
\int_{X} h_{2} f_{k} d \mu & \int_{X} h_{2}^{2} d \mu & \cdots & \int_{X} h_{2} h_{n} d \mu \\
\vdots & \vdots & \ddots & \vdots \\
\int_{X} h_{n} f_{k} d \mu & \int_{X} h_{n} h_{2} d \mu & \cdots & \int_{X} h_{n}^{2} d \mu
\end{array}\right)\right|
\end{array}
$$

for all $h_{2}, \ldots, h_{n} \in L^{2}(X, \mu)$. 
If, in addition,

$$
\begin{array}{r}
\lim _{k \rightarrow \infty}\left|\left(\begin{array}{cccc}
\int_{X} f_{k}^{2} d \mu & \int_{X} f_{k} h_{2} d \mu & \cdots & \int_{X} f_{k} h_{n} d \mu \\
\int_{X} h_{2} f_{k} d \mu & \int_{X} h_{2}^{2} d \mu & \cdots & \int_{X} h_{2} h_{n} d \mu \\
\vdots & \vdots & \ddots & \vdots \\
\int_{X} h_{n} f_{k} d \mu & \int_{X} h_{n} h_{2} d \mu & \cdots & \int_{X} h_{n}^{2} d \mu
\end{array}\right)\right| \\
=\left|\left(\begin{array}{cccc}
\int_{X} f^{2} d \mu & \int_{X} f h_{2} d \mu & \cdots & \int_{X} f h_{n} d \mu \\
\int_{X} h_{2} f d \mu & \int_{X} h_{2}^{2} d \mu & \cdots & \int_{X} h_{2} h_{n} d \mu \\
\vdots & \vdots & \ddots & \vdots \\
\int_{X} h_{n} f d \mu & \int_{X} h_{n} h_{2} d \mu & \cdots & \int_{X} h_{n}^{2} d \mu
\end{array}\right)\right|
\end{array}
$$

for all $h_{2}, \ldots, h_{n} \in L^{2}(X, \mu)$, then

$$
\lim _{k \rightarrow \infty}\left|\left(\begin{array}{cccc}
\int_{X}\left(f_{k}-f\right)^{2} d \mu & \int_{X}\left(f_{k}-f\right) h_{2} d \mu & \cdots & \int_{X}\left(f_{k}-f\right) h_{n} d \mu \\
\int_{X} h_{2}\left(f_{k}-f\right) d \mu & \int_{X} h_{2}^{2} d \mu & \cdots & \int_{X} h_{2} h_{n} d \mu \\
\vdots & \vdots & \ddots & \vdots \\
\int_{X} h_{n}\left(f_{k}-f\right) d \mu & \int_{X} h_{n} h_{2} d \mu & \cdots & \int_{X} h_{n}^{2} d \mu
\end{array}\right)\right|=0
$$

for all $h_{2}, \ldots, h_{n} \in L^{2}(X, \mu)$.

Proof. This follows from Theorem 2.12.

Proposition 3.3. Let $f_{k} \in L^{2}(X, \mu), k=1,2, \ldots$, be such that

$$
\lim _{k \rightarrow \infty}\left|\left(\begin{array}{cccc}
\int_{X} f_{k} g d \mu & \int_{X} f_{k} h_{2} d \mu & \cdots & \int_{X} f_{k} h_{n} d \mu \\
\int_{X} h_{2} g d \mu & \int_{X} h_{2}^{2} d \mu & \cdots & \int_{X} h_{2} h_{n} d \mu \\
\vdots & \vdots & \ddots & \vdots \\
\int_{X} h_{n} g d \mu & \int_{X} h_{n} h_{2} d \mu & \cdots & \int_{X} h_{n}^{2} d \mu
\end{array}\right)\right|=0
$$

for all $g, h_{2}, \ldots, h_{n} \in L^{2}(X, \mu)$, and there exists a constant $M$ such that

$$
\lim _{m \rightarrow \infty} \frac{1}{m^{2}} \sum_{k=1}^{m}\left|\left(\begin{array}{cccc}
\int_{X} f_{k}^{2} d \mu & \int_{X} f_{k} h_{2} d \mu & \cdots & \int_{X} f_{k} h_{n} d \mu \\
\int_{X} h_{2} f_{k} d \mu & \int_{X} h_{2}^{2} d \mu & \cdots & \int_{X} h_{2} h_{n} d \mu \\
\vdots & \vdots & \ddots & \vdots \\
\int_{X} h_{n} f_{k} d \mu & \int_{X} h_{n} h_{2} d \mu & \cdots & \int_{X} h_{n}^{2} d \mu
\end{array}\right)\right|=0
$$

for all $h_{2}, \ldots, h_{n} \in L^{2}(X, \mu)$. Then there exists a sequence $\left(g_{k}\right)$ of finite convex combinations of $\left(f_{k}\right)$, such that

$$
\lim _{k \rightarrow \infty}\left|\left(\begin{array}{cccc}
\int_{X} g_{k}^{2} d \mu & \int_{X} g_{k} h_{2} d \mu & \cdots & \int_{X} g_{k} h_{n} d \mu \\
\int_{X} h_{2} g_{k} d \mu & \int_{X} h_{2}^{2} d \mu & \cdots & \int_{X} h_{2} h_{n} d \mu \\
\vdots & \vdots & \ddots & \vdots \\
\int_{X} h_{n} g_{k} d \mu & \int_{X} h_{n} h_{2} d \mu & \cdots & \int_{X} h_{n}^{2} d \mu
\end{array}\right)\right|=0
$$

for all $h_{2}, \ldots, h_{n} \in L^{2}(X, \mu)$.

Proof. This follows from Theorem 2.13. 
Remark 3.4. Note that we also have another equivalent formula for the standard $n$-inner product and $n$-norm in $L^{2}(X)$ as follows (see $[3,9]$ )

$$
\left\langle f, g \mid h_{2}, \ldots, h_{n}\right\rangle=\frac{1}{n !} \underbrace{\int_{X} \int_{X} \ldots \int_{X}}_{n \text { times }} \operatorname{det}(F) \operatorname{det}(G) d \mu\left(x_{1}\right) \ldots d \mu\left(x_{n}\right)
$$

where

$$
\operatorname{det}(F)=\left|\left(\begin{array}{cccc}
f\left(x_{1}\right) & f\left(x_{2}\right) & \cdots & f\left(x_{n}\right) \\
h_{2}\left(x_{1}\right) & h_{2}\left(x_{2}\right) & \cdots & h_{2}\left(x_{n}\right) \\
\vdots & \vdots & \ddots & \vdots \\
h_{n}\left(x_{1}\right) & h_{n}\left(x_{2}\right) & \cdots & h_{n}\left(x_{n}\right)
\end{array}\right)\right|
$$

and

$$
\operatorname{det}(G)=\left|\left(\begin{array}{cccc}
g\left(x_{1}\right) & g\left(x_{2}\right) & \cdots & g\left(x_{n}\right) \\
h_{2}\left(x_{1}\right) & h_{2}\left(x_{2}\right) & \cdots & h_{2}\left(x_{n}\right) \\
\vdots & \vdots & \ddots & \vdots \\
h_{n}\left(x_{1}\right) & h_{n}\left(x_{2}\right) & \cdots & h_{n}\left(x_{n}\right)
\end{array}\right)\right|
$$

The standard $n$-norm is therefore

$$
\left\|f, h_{2}, \ldots, h_{n}\right\|=(\frac{1}{n !} \underbrace{\int_{X} \int_{X} \ldots \int_{X}}_{n \text { times }}[\operatorname{det}(F)]^{2} d \mu\left(x_{1}\right) \ldots d \mu\left(x_{n}\right))^{1 / 2}
$$

The above propositions hold accordingly using this form of $n$-inner product and n-norm.

Similarly, we can get other results concerning weak and strong convergence in other $n$-Hilbert spaces. For instance, we mention the Sobolev space $W^{s, 2}(\Omega)=$ $H^{s}(\Omega)$, which is a Hilbert space with inner product

$$
\langle f, g\rangle=\int_{\Omega} f(x) g(x) d x+\sum_{i=1}^{s} \int_{\Omega} D^{i} f(x) \cdot D^{i} g(x) d x
$$

We can equip $H^{s}(\Omega)$ with the standard $n$-inner product (1), and all the above convergence results will hold accordingly.

\section{REFERENCES}

1. Chu, H.Y., Park, C., Park, W., "The Aleksandrov problem in linear 2-normed spaces", J. Math. Anal. Appl. 289 (2004), 666-672.

2. Chu, H.Y., Ku, S.H., Kang, D.S., "Characterizations on 2-isometries", J. Math. Anal. Appl. 340 (2008), 621-628.

3. Chugh, R., Lather, S., "On reverses of some inequalities in $n$-inner product spaces", Int. J. Math. Math. Sci. (2010), Article ID 385824

4. Gähler, S., "Lineare 2-normietre räume", Math. Nachr. 28 (1965), 1-43.

5. Gozali, S.M., Gunawan, H., Neswan, O., "On $n$-norms and $n$-bounded linear functionals in a Hilbert space", Ann. Funct. Anal. 1 (2010), 72-79. 
6. Gunawan, H., "On convergence in $n$-inner product spaces", Bull. Malays. Math. Sci. Soc. (2) 25 (2002), 11-16.

7. Gunawan, H., "A generalization of Bessel's inequality and Parseval's identity", Per. Math. Hungar. (2002), 177-181.

8. Gunawan, H., Mashadi, "On n-normed spaces", Int. J. Math. Math. Sci. 27 (2001), 631-639.

9. Gunawan, H., "The space of $p$-summable sequences and its natural $n$-norms", Bull. Australian Math. Soc. 64 (2001), 137-147.

10. Misiak, A., "n-inner product spaces", Math. Nachr. 140 (1989), 299-319.

11. Soenjaya, A.L., "On $n$-bounded and $n$-continuous operator in $n$-normed space", J. Indonesian Math. Soc. Vol 18 No. 1 (2012), 45-56.

12. White, A., "2-Banach spaces", Math. Nachr. 42 (1969), 43-60. 